\title{
Complexity of the Self-Schema and Responses to Disconfirming Feedback
}

\author{
Karen Farchaus Stein ${ }^{1}$ \\ University of Michigan
}

This study focused on complexity of the self-schema as one factor that influences people's responses to social feedback that challenges their established view of self. Complexity refers to the number of independent attributes included in the schema. A card-sorting task (Zajonc, 1960) was used to identify the high- and low-complexity groups. Subjects were given bogus feedback relevant to the targeted domain of self-knowledge, and changes in self-descriptiveness ratings and response latency times were monitored. Results suggest that high-complexity subjects were able to attend to and encode the disconfirming feedback, while low-complexity subjects responded by rejecting the feedback and reasserting positive aspects of the self. The implications of these findings for clarifying the process of self-schema updating, revision, and change are discussed.

KEY WORDS: self-complexity; self-schema change; self-schema; information processing.

In the last decade, empirical work on the self-concept has tended to emphasize its stable and enduring nature. Studies have shown that people not only prefer social feedback that is consistent with their view of themselves (Swann \& Read, 1981) but, in addition, tend to ignore or reject disconfirming social feedback rather than change the established self-view (Greenwald, 1980; Greenwald \& Pratkanis, 1984; Markus, 1977). Together these findings have led investigators to conclude that the self is a relatively immutable cognitive structure-one that resists new and threatening information from the social environment.

${ }^{1}$ Address all correspondence to Karen Stein, $400 \mathrm{~N}$. Ingalls, Room 2344, University of Michigan, Ann Arbor, Michigan 48109-0482. 
The current emphasis on stability of the self-structure, however, has directed attention away from the fact that people must and, in fact, often do use social feedback to update or revise the established self-view. Consider, for example, the high school valedictorian who adjusts her "naturally smart and effortlessly successful" self-view to accommodate her mediocre first college semester grades or the type-A businessman who modifies his "healthy and invulnerable" self-conception to include the recent news that he is hypertensive. These examples suggest that the self cannot realistically be viewed as a closed system that uniformly resists or rejects all disconfirming feedback. In fact, even those who ardently support the conceptualization of the self as a stable and enduring cognitive structure acknowledge that the maintenance of a relevant and realistic view of the self is dependent on the person's ability to use social feedback to update or revise the self-view (see Swann \& Read, 1981).

Yet despite the fact that the utilization of social feedback is central to the maintenance of a current and realistic self-view, the recent emphasis on the stability of the self-structure has meant that little empirical attention has been directed toward understanding the factors that enable people to consider and use social feedback. In this paper properties of the self-structure that play a role in shaping the individual's response to disconfirming social feedback are explored. Building on empirical work that examined the effects of cognitive complexity on the utilization of contradictory social information, it is posited that people with highly complex self-schemas may be more able to consider and use disconfirming feedback to update their self-view than those with less complex self-schemas. Furthermore, it is argued that previous work that has highlighted the stable and resistant nature of the self-structure has focused on changes in global self-defining attributes rather than exploring more subtle process measures that might reflect the person's ability to consider and encode the new and contradictory selfrelevant information.

\section{Cognitive Complexity}

The concept of cognitive complexity was derived from Werner's developmental theory and Kelly's theory of personal constructs (Bieri, 1955; Burleson, 1987; Crockett, 1965). It is based on the assumption that people represent objects in memory as systems of bidirectional dimensions or constructs. Although the original conceptualization of complexity focused both on the number of constructs within the cognition and the extent to which the constructs were hierarchically organized (for example, see Crockett, 1965), recent definitions focus exclusively on the quantity of information 
(Burleson, 1987). Complexity is generally defined as the number of distinct or independent attributes included in the cognitive structure (Bieri, 1955; Linville, 1985, 1987; O'Keefe \& Sypher, 1981; Scott, Osgood, \& Peterson, 1979). A highly complex cognition is one that includes many distinct units of information, while a less complex structure consists of relatively fewer distinct units.

Linville (1985) examined the effects of complexity of the self-concept on stability of affect and self-esteem. This study focused on the total array of knowledge about the self available in memory. Individuals with high complexity and those with low complexity of the total self-concept were given positive or negative feedback about their performance on a cognitive task. The findings revealed that low-complexity subjects responded to the feedback with more extreme changes in mood and self-esteem in the direction consistent with the feedback than did high-complexity subjects. Based on the data, one can conclude that low-complexity subjects tend to focus on and react to a single perspective of the self while apparently losing sight of other available self-conceptions.

Although the findings of Linville's (1985) study provided evidence to suggest that complexity of the self-concept plays an important role in the processing of incongruent information, many important questions remain unanswered. Do the differences in emotional reactivity reflect differences in ability to attend to and utilize the new information? Does the emotional reactivity of low-complexity subjects mean that they are more likely to take in and encode the new information? Do high-complexity subjects maintain stability in affect and self-esteem by blocking out or ignoring disconfirming feedback? Or does the rich, highly complex structure enable the individual to incorporate the new information while maintaining the established selfview?

\section{Cognitive Complexity and Bivalent Information}

The findings of a series of studies on impression formation offer further insight into the effects of cognitive complexity on the processing of incongruent information. In these studies investigators found that individuals with highly elaborated cognitive structures of other people were more able to consider and use contradictory information to form an integrated understanding of another person than those with less elaborated structures (Crockett, 1965; Leventhal, 1957; Mayo \& Crockett, 1964). Using the typical impression formation paradigm, subjects were presented with a collection of positive and negative descriptors of a target person. High-complexity subjects were able to attend to and use bivalent information to form an 
impression of the target that was consistent with the information provided. In contrast, low-complexity subjects used only one cluster of traits, either those presented first in the description (i.e., primacy effect) or those presented last (i.e., recency effect), while ignoring the other conflicting traits.

Two different but highly related explanations of the mechanisms underlying the relationship between complexity of the cognitive structure and responses to bivalent information have been offered. The first explanation is based on the argument that differentiation and hierarchical organization of the cognition are cooccurring, such that as a cognition becomes more highly differentiated, it necessarily becomes more hierarchically organized. As the hierarchical structuring becomes more elaborated, the person has available in memory a collection of superordinate abstractions that can be used to reconcile disparate behaviors or traits (Crockett, 1965; Mayo \& Crockett, 1964; Metlzer, Crockett, \& Rosenkrantz, 1966). A second explanation focuses simply on the amount of information included in the cognitive structure. According to this view the availability of many distinct units of information enables more flexible processing of information (Bieri, 1955; Burleson, 1987; Scott et al., 1979).

\section{Complexity of the Self-Schema and Processing Disconfirming Feedback}

Based on the findings from the impression formation literature, it is posited that the availability of a highly complex self-schema will enable the individual to attend to and incorporate social feedback that is incongruent with the generalized view of the self in the domain. In contrast, when a schema is less complex, the individual has a smaller and less diverse array of information available in memory that can be brought to bear in processing the new and contradictory information. In this case the individual has less flexibility in thinking about the self and, consequently, may have more difficulty attending to and utilizing the new and contradictory information.

The study that follows was designed as a preliminary test of this hypothesis. However, unlike previous studies which focused on changes in self-ratings on trait adjectives as a primary indicator of the person's acceptance or rejection of the disconfirming feedback, this study was founded on the idea that response latency times for the self-rating judgments may be a more sensitive measure of whether the new information was encoded in memory.

The focus on response latency is based on the view that self-schemas are hierarchical organizations of knowledge in which situation specific images of the self are nested within higher order trait abstractions (see Hastie, 
1981; Markus \& Sentis, 1982; Taylor \& Crocker, 1981). Typically, judgments about the self in the domain are made quickly and efficiently by comparing the stimulus to a higher-level trait abstraction. However, under certain circumstances, the lower-level situation-specific memories are evoked. The use of this rich, detailed collection of memories as the processing framework leads to longer response latency time for self-descriptiveness judgments (Lewicki, 1986; Taylor \& Crocker, 1981).

Based on this model of the self-schema, it is argued that a piece of disconfirming feedback about the self will be encoded as a situation-specific memory at a lower level in the hierarchical structure. The recent addition of an evaluatively incongruent piece of information may make the situation-specific memories more readily accessible and, at least temporarily, increase the likelihood that they will be evoked during subsequent judgments about the self. Consequently, the process of making the judgment about the self-descriptiveness of a stimulus is more complicated and, hence, time-consuming as this rich and evaluatively mixed collection of situationspecific memories is scanned to determine the degree of congruence between the stimulus and the self (Lewicki, 1986).

The encoding of evaluatively inconsistent feedback would not, however, be expected to cause a measurable change in the content of subsequent judgments about the self. Because the feedback is encoded as one of many situation-specific memories in the domain, its power to influence subsequent judgments will be diluted and, therefore, minimal. Although the task of evaluating the congruence between the stimulus and the self becomes more complicated, it is likely that the match between the stimulus and the self will be determined by the majority of memories in the domain rather than by any single instance.

If on the other hand, the new information is ignored or rejected and, therefore, not encoded within schema, the cognitive framework underlying the judgment process will remain unaltered. In this case the higher-order trait abstraction is likely to continue to serve as the framework for the judgment, and the processing time for relevant self-evaluative judgments made after receiving the feedback should remain unchanged.

\section{METHOD}

An initial step in preparing for this study was to identify a group of individuals who had in common a domain of self-knowledge and to measure the complexity of that self-schema. The "student" self-schema in an undergraduate college population was chosen because it was assumed that all students would have some established conceptions of themselves in the role 
and that these self-conceptions would generally be highly valued and centrally self-defining.

During the first of two experimental sessions, complexity of the student self-schema and self-descriptiveness ratings of the self in the domain were measured. The self-descriptiveness ratings were computerized, and unbeknownst to the subject, response latency times for the ratings were also measured. At the completion of the session subjects were asked by a different experimenter to participate in an ostensibly unrelated study designed to validate a new intelligence test.

During the second experimental session approximately 2 weeks later, subjects were asked to complete a fictitious intelligence test and were given bogus feedback about their performance. Self-descriptiveness ratings were measured again after the feedback was given, enabling the amount of change in response latency times and self-descriptiveness ratings caused by the feedback to be determined.

\section{Subjects}

For session 1, subjects were 151 male and female undergraduate students. Subjects were recruited from classes throughout the University and were paid $\$ 10$ for their participation. One hundred nineteen of the original 151 subjects $(79 \%)$ completed session $2 .^{2}$ Of those 119 subjects, $63 \%(n=$ $75)$ were female and $37 \%(n=44)$ were male. Subjects were paid an additional $\$ 5$ for session 2 .

\section{Session 1}

Procedure and measures. For the first laboratory session, subjects were run in small groups comprised of one to four subjects. Subjects were told by the experimenter that the purpose of the study was to learn about individual differences in the way people think and feel about themselves. The experimental session took approximately $1 \frac{1}{2}$ hours to complete. During this session subjects completed the following measures:

${ }^{2}$ Subjects who agreed to participate in session 2 did not differ significantly from those who refused to participate in differentiation scores (participants: $M=19.1, S D=5.5$; nonparticipants: $M=19.9, S D=5.4, t<1$ ) nor in unity scores (participants: $M=0.265$, $S D=0.109$; nonparticipants: $M=0.262, S D=0.125, t<1$ ). The two groups also did not differ in baseline measures of self-descriptiveness ratings (participants: $M=8.9, S D=1.3$; nonparticipants: $M=9.0, S D=1.0, t<1$ ) nor in baseline response latency time for the self-descriptiveness ratings (participants: $M=5.04 \mathrm{sec}, S D=1.31$; nonparticipants: $M=$ 5.16, $S D=1.49, t<1$ ) suggesting that motivation to participate was not related to the independent or dependent variables. 
1. Complexity of the self-schema. A card-sorting method for measuring the organizational properties of cognitive structures developed by Zajonc $(1954,1960)$ was used to measure complexity of the student self-schema. For this measure subjects were first given a stack of 52 blank index cards which were marked from $\mathrm{A}$ to $\mathrm{ZZ}$. The subjects were then instructed:

Now I would like you to think of yourself in the student role. On each card separately write one characteristic which describes you as a student. You can put down whatever comes to your mind, since there is no one list of characteristics that can be considered as either "correct" or "incorrect." Everyone's thoughts about themselves are different.

Write down as many characteristics as you feel are necessary to describe yourself as a student. You may have too many or too few cards, but this shouldn't bother you. If you find that you need additional cards raise your hand and I will bring extra cards to your seat. Work rapidly.

Next subjects were instructed:

It is possible that some of the characteristics you identified are related to one another. They may depend on one another in such a way that if one changes, the other one would change too. Suppose the table in front of you was bigger than it is now. Then it would become heavier. This means that the weight of the table depends on the size. The relationships between characteristics you put down may not be so obvious and so simple, but try to decide whether such relationships exist, nevertheless. To do this lay out the cards in front of you in alphabetical order, and list all the characteristics which would change if Characteristic A were changed, absent or untrue of you; list all the characteristics that would change if Characteristic B were changed, absent or untrue of you, etc.

The responses to these tasks were used to identify subjects with high complexity and those with low complexity of the student self-schema.

In this study complexity refers to two properties of the self-schema: (1) differentiation - the number of attributes included in the self-schema and (2) unity - the degree of dependence among the attributes included in the schema. High complexity refers to self-schemas that have many independent attributes included in the structure, whereas low complexity refers to selfschemas that have few attributes that are highly interdependent.

A differentiation score was computed by counting the number of characteristics generated during the first task. To calculate a unity score, the responses to the second task were used to form a dependency matrix such that when $A_{j}$ caused a change in $A_{i}$ a value of 1 was assigned. The total dependency of an element was calculated by summing the row entries, and the total dependency of the schema was calculated by summing the dependencies across all characteristics. To compare the degree of unity across schemas with varying levels of differentiation, it is necessary to normalize the measure of unity by dividing the total dependence of the schema by the total number of possible dependencies of the schema: 


$$
\text { Unity }=\frac{\sum_{i=1}^{n} \text { dependency }\left(A_{i}\right)}{n(n-1)}
$$

2. Domain specific self-descriptiveness measure. The domain-specific self-descriptiveness measure consisted of 38 semantic differential scales. Thirty-five scales measured the individual's view of the self in the student domain; 25 scales focused on performance aspects of the student role (e.g., competent-incompetent, intelligent-unintelligent), and 10 focused on the social aspects of the student role. Three practice items were also included.

Each bipolar set of adjectives was presented on a 12-point scale. The positive and negative self-descriptors were alternately presented on the right side of the scale. The scales were presented on a cathode ray tube screen (CRT) in four randomly determined orders of presentation. Each item appeared on CRT for a maximum of $30 \mathrm{sec}$. There was a 1-sec interval between the subject's response and the presentation of the next scale. Subjects were asked to key in the number that best described them on the dimension. Response latency times (interval between when the scale first appeared on the screen and the moment when the first key was struck) were also recorded. Subjects were unaware that response latency times were being measured.

A total student self-descriptiveness score was determined for each subject by computing a mean of 23 performance-related student scales. Of the original 25 performance scales, two were deleted to increase the internal consistency of the total measure to an alpha coefficient of .87 . For this measure scores were computed such that the higher the score, the more positive the self-descriptive ratings in the student domain.

3. Importance of behavioral domains. After the self-descriptiveness measure was completed, subjects were asked to rate six behavioral domains (e.g., friend, athlete, student) according to the importance of each domain to their overall view of themselves. Each domain was presented on a 12point scale, ranging from extremely important to extremely unimportant. High scores on this measure reflect high importance of the domain.

4. Rosenberg Self-Esteem Scale (RSES; Rosenberg, 1965). The RSES was used to measure the individual's global self-satisfaction. The RSES is a 10-item Guttman scale (e.g., "I have a number of good qualities"). Item values (ranging from 1 to 4 ) are summed to compute the self-esteem score. High scores reflect positive self-esteem.

5. Written fluency. To examine the effects of individual differences in ability and motivation to communicate thoughts in writing on the differen- 
tiation and unity scores, a measure of written fluency was completed. Subjects were shown a $5 \times 7$-in black and white photograph of a young couple walking down the street and were given 7 min to write a story about the picture. A written fluency score was computed by counting the number of words written.

6. Intelligence Measure. At the end of Session 1 subjects were asked to report either their college entry Scholastic Aptitude Total scores (SAT) or their American College Test scores (ACT) as an indicator of intelligence.

\section{Session 2}

At the completion of the first experimental session, subjects were approached by a second experimenter ostensibly looking for volunteers to participate in a study designed to test and validate an intelligence test. The names and phone numbers of subjects who were willing to participate in the study were taken. Approximately 2 weeks later the experimenter contacted each subject by phone and a time for the experimental session was scheduled.

For this session, subjects were run individually. At the beginning of session 2, the subject was asked to complete the Michigan Conceptual Intelligence Test, which was actually a modified version of the Quick Test of Intelligence (Ammons \& Ammons, 1962). When the test was completed, the experimenter left the room for several minutes after she explained that she would enter the subject's responses into the computer for scoring. The experimenter returned to the room several minutes later with a computer printout that gave a raw score and a percentile ranking. Subjects were randomly assigned to either the success condition, in which they were told that they scored in the top $3 \%$, or the failure condition, in which they were told that they scored in the bottom $47 \%$ of college students who had completed the test to date.

After subjects received the feedback, the experimenter told them that in order to validate the test and verify the norms, it was necessary for her to collect information about their personal backgrounds. She explained that the background information questionnaire was computerized so that subjects would be asked to enter their responses at a computer terminal. She then left the room after she stated that she would check to make sure that the computer was ready. The experimenter left the room without providing an opportunity for the subject to discuss his/her score. She returned after a 5-min interval and the subjects then completed a demographic data ques- 
tionnaire and the self-descriptiveness measure. ${ }^{3}$ At the completion of session 2 , all subjects were fully debriefed and thanked for their participation.

\section{RESULTS}

\section{Organizational Properties of the Student Self-Schema}

As expected, the student domain of self-knowledge was generally considered highly important to the participants' overall views of themselves ( $M=10.09, S D=1.79$, on a 12-point scale). However, despite the overall importance of this domain of self-knowledge, considerable variability in the organizational properties of the self-schema was found. For the total sample, the mean differentiation score (the number of characteristics included in the schema) was 19.3 (range $=10-34$ ) and the mean unity score (the degree of dependence among the attributes included in the schema) was 0.26 (range $=0.07$ to 0.62 ; possible range $=0$ to 1.0 where 1.0 is total dependency among the attributes).

In the total sample, differentiation and unity were moderately and negatively correlated, $r(135)=-.30, p<.001$. In this domain of self-knowledge, schemas that were highly elaborated or had many characteristics included within them tended to have more independence among the attributes that schemas that included fewer characteristics.

\section{Identification of High- and Low-Complexity Groups}

For the purpose of examining the relationship between complexity of the self-schema and ability to attend to and incorporate disconfirming feedback, two groups of subjects were identified: individuals with high complexity of the student self-schema and individuals with low complexity of the student self-schema. Complexity is a combined measure that refers to both the number of attributes included in the schema and the amount of interdependence among the attributes. Placement in the high- and lowcomplexity groups was determined by the differentiation and unity scores derived from the card-sorting measure.

A median split was used to identify high and low differentiation subjects and high- and low-unity subjects. A cross tabulation was then used to

${ }^{3}$ The 10 social items and the three practice items were deleted from the time 2 self-descriptiveness measure to decrease the similarity between the time 1 and time 2 measures and, thereby, reduce the likelihood that subjects would recognize the association between the two experimental sessions. 
identify subjects who had high differentiation scores and low unity scores, the high complexity group $(n=34)$ and those who had low differentiation scores and high unity scores, the low complexity group $(n=37){ }^{4}$

\section{Equivalence of Groups}

One assumption underlying this experimental manipulation was that most subjects in this population would have a highly positive self-schema established in the student domain and, therefore, the negative feedback would be experienced as a threat to the self. To test this assumption, baseline levels of the student self-descriptiveness ratings were examined. For the high- and low-complexity groups combined, the mean self-descriptiveness rating was $9.0(S D=1.4)$ on a 12-point scale, suggesting that, in general, subjects viewed themselves as above-average students. Group comparisons revealed that the high-complexity group $(M=9.0, S D=1.4)$ and the low-complexity group $(M=8.9, S D=1.4)$ had very positive and approximately equal self-descriptiveness ratings at time $1, t<1$. The two groups also had high and approximately equal baseline levels of global selfesteem as measured by the RSES (high-complexity $M=31.4, S D=5.1$; low-complexity $M=31.3, S D=5.2 ; t<1$ ).

To examine the effects of ability and motivation to communicate one's thoughts in writing and intelligence on group placement, written fluency, SAT and ACT scores were compared between the two groups. No significant differences were found (all $t<1$ ).

No significant differences were found between the two groups in time 1 response latency scores (high-complexity $M=4.66, S D=1.24$; low-complexity $M=5.12, S D=1.34, t=1.49, p=.14$ ). In addition, no gender differences were found in the independent nor dependent variables, and, therefore, gender was not considered in any of the subsequent analyses.

\footnotetext{
${ }^{4}$ The decision to treat complexity as a single dichotomous variable is based on the theoretical view that processing differences occur after the cognitive structure reaches some critical level of complexity rather than expecting continuous processing differences with each incremental increase in the number of independent conceptions included in the structure. In other words, it is postulated that some critical number of independent conceptions within the self-schema is necessary before the individual is able to encode disconfirming information. Until the critical number of self-conceptions is available, individuals will have difficulty processing the disconfirming information. This view is consistent with most previous research on cognitive complexity.
} 


\section{Response Latency Times for Self-Descriptiveness Judgments}

The primary hypothesis guiding this study was that high-complexity subjects would be more likely than low-complexity subjects to attend to and encode the disconfirming feedback. Because of the availability in memory of this new information, high-complexity subjects were expected to be slower to make relevant self-descriptiveness judgments after receiving the failure feedback than the low-complexity group. Furthermore, because the success feedback was consistent with the sample's highly positive self-view in the student domain, the same differences in response latency times were not expected between the high- and low-complexity subjects in the success condition.

To test this hypothesis an analysis of covariance (ANCOVA) model was used to determine how response latency times for the self-descriptiveness judgments varied with complexity of the student self-schema and feedback condition. The dependent variable for this analysis was the mean response latency change score. This score was calculated by subtracting the time 1 mean response latency score for the student self-descriptiveness judgments from the time 2 score. For the response latency change score, the larger the negative number, the faster the subject was relative to time 1. The time 1 mean response latency score was used as the covariate and condition (success/failure) and complexity (high/low) were included in the model as independent variables.

Results of the covariance analysis revealed an expected significant relationship between the covariate, time 1 response latency score, and the dependent measure, the response latency change score, $F(1,70)=47.61$, $p<.001$, indicating that, the slower the subject was at time 1 , the more change there was between time 1 and time 2 . A significant main effect for complexity was also found, suggesting that high-complexity subjects experienced less change between time 1 and time 2 response latency scores $(M$ $=-0.41 \mathrm{sec})$ than the low-complexity group $(M=-0.98 \mathrm{sec})$ regardless of

Table I. Mean Response Latency Scores (Standard Deviations in Parentheses) for Lowand High-Complexity Subjects in the Failure and Success Conditions

\begin{tabular}{lcccrr}
\hline & \multicolumn{2}{c}{ Failure } & & \multicolumn{2}{c}{ Success } \\
\cline { 2 - 3 } \cline { 5 - 6 } $\begin{array}{l}\text { Response latency } \\
\text { scores }(\mathrm{sec})\end{array}$ & $\begin{array}{c}\text { Low } \\
\text { complexity }\end{array}$ & $\begin{array}{c}\text { High } \\
\text { complexity }\end{array}$ & & $\begin{array}{c}\text { Low } \\
\text { complexity }\end{array}$ & $\begin{array}{c}\text { High } \\
\text { complexity }\end{array}$ \\
\hline Time 1 & $4.88(1.29)$ & $4.59(1.09)$ & & $5.33(1.38)$ & $4.71(1.35)$ \\
Time 2 & $3.71(0.89)$ & $4.54(0.98)$ & & $4.51(1.02)$ & $4.08(1.13)$ \\
Change score $^{a}$ & $-1.17(0.80)$ & $-0.05(1.20)$ & & $-0.82(0.83)$ & $-0.63(0.97)$ \\
\hline
\end{tabular}

${ }^{a}$ For the response latency change scores, the larger the negative number, the faster subjects were relative to time 1 . 
the feedback condition, $F(1,70)=4.19, p<.05$. Also as predicted, a significant two-way interaction between complexity and condition was found, $F(1,70)=9.16, p=.004$.

Planned contrasts shown in Table I revealed that, in the failure condition, high-complexity subjects experienced significantly less change in response latency scores between time 1 and time 2 than the low-complexity group, $t(28)=3.06, p=.005$. In the success condition, no significant difference in response latency change scores was found between the highand low-complexity subjects, $t(39)<1$.

In addition, no significant differences in mean response latency change scores were found between the low-complexity subjects in the success and failure conditions. However, high-complexity subjects in the failure condition tended to experience less change between time 1 and time 2 response latency scores than the high-complexity subjects in the success condition, but this difference failed to reach significance, $t(32)=-1.54, p=.13$.

Analysis of covariance using the time 1 response latency score as the covariate and the time 2 response latency score as the dependent variable revealed the same pattern of effects. ${ }^{5}$

\section{Self-Descriptiveness Ratings}

An analysis of covariance (ANCOVA) was also used to determine how changes in self-descriptiveness judgments varied with complexity of the student self-schema and feedback condition. The dependent variable for this analysis was the mean self-descriptiveness change score (time 2 mean self-descriptiveness score minus time 1 mean self-descriptiveness score). For the mean self-descriptiveness change score, the larger the positive number, the more positive the self-rating was at time 2 compared to time 1 . The time 1 mean self-descriptiveness score was used as the covariate, and condition (success/failure) and complexity (high/low) were entered as independent variables.

\footnotetext{
${ }^{5}$ Most past research has treated complexity as a dichotomous variable. To find out whether the results depended on using complexity as dichotomy, a regression analysis was conducted to examine the relationship of response latency to complexity and condition, when complexity was represented as a continuous variable. For this analysis, a continuous measure of complexity was constructed giving equal weight to its two components by the following steps: (a) Unity was reverse-scored, indicating independence, (b) the differentiation and independence scores were transformed to $z$-scores, and the $z$-scores were added together. In the analysis, response latency change scores were regressed on time 1 latency, the continuous complexity score, condition (entered as a dummy variable), and the product of complexity and condition. This analysis found a significant interaction between the complexity and condition term, $t(102)=2.13, p=.036$, of the same form as shown when complexity was treated as a dichotomy.
} 
The results of the covariance analysis revealed an expected significant relationship between the covariate, the time 1 self-descriptiveness score and the dependent measure, self-descriptiveness change score, $F(1,70)=9.90$, $p=.002$, indicating that the lower the self-descriptiveness rating at time 1 , the more change there was between the time 1 and time 2 scores. In addition, a significant main effect for complexity was found, $F(1,70)=$ $5.17, p<.03$, suggesting that high-complexity subjects experienced less change in their self-descriptiveness scores between time 1 and time 2 than the low-complexity subjects, regardless of the feedback condition. The twoway interaction effect between condition and complexity failed to reach significance, $F(1,70)=1.96, p<.17$.

Although a significant two-way interaction was not found, the planned two-group comparisons were completed. As shown in Table II, there is a trend in the data suggesting that low-complexity subjects experienced a greater increase in self-descriptiveness scores after receiving the failure feedback than high-complexity subjects in the same condition $t(28)=2.94$, $p=.007$. No differences in mean self-descriptiveness change scores were found between the high- and low-complexity subjects in the success condition. Similarly, no differences were found between the low-complexity subjects in the failure and success conditions nor between the high-complexity groups in the failure and success conditions. ${ }^{6}$

\section{DISCUSSION}

The findings of this study lend preliminary support to the hypothesis that complexity of the self-schema plays an important role in shaping the

Table II. Mean Self-Descriptiveness Scores (Standard Deviations in Parentheses) for Lowand High-Complexity Subjects in the Failure and Success Conditions

\begin{tabular}{|c|c|c|c|c|}
\hline \multirow{2}{*}{$\begin{array}{c}\text { Self- } \\
\text { descriptiveness } \\
\text { scores }\end{array}$} & \multicolumn{2}{|c|}{ Failure } & \multicolumn{2}{|c|}{ Success } \\
\hline & $\begin{array}{c}\text { Low } \\
\text { complexity }\end{array}$ & $\begin{array}{c}\text { High } \\
\text { complexity }\end{array}$ & $\begin{array}{c}\text { Low } \\
\text { complexity }\end{array}$ & $\begin{array}{c}\text { High } \\
\text { complexity }\end{array}$ \\
\hline Time 1 & $8.67(1.52)$ & $8.95(1.86)$ & $9.14(1.34)$ & $9.06(1.07)$ \\
\hline Time 2 & $9.22(1.46)$ & $8.87(1.70)$ & $9.42(1.28)$ & $9.19(1.05)$ \\
\hline Change score ${ }^{a}$ & $0.55(0.48)$ & $-0.08(0.70)$ & $0.29(0.78)$ & $0.14(0.64)$ \\
\hline
\end{tabular}

${ }^{a}$ For the self-descriptiveness change scores, the larger the positive number, the more positive the self-rating was at time 2 compared to time 1 .

${ }^{6} \mathrm{~A}$ regression analysis was also used to examine the relationship between complexity and condition and the mean self-descriptiveness change score. No reliable relationships were detected. 
person's response to social feedback that challenges the established selfview. As expected, the response latency times for the self-descriptiveness judgments provided valuable information about the processing differences between high- and low-complexity subjects. The fact that all groups had faster response latency times at time 2 is probably attributable to familiarity with the measurement technique. Yet despite the fact that all groups were equally familiar with the technique at time 2 , high-complexity subjects in the failure condition sped up less at time 2 than the low-complexity subjects in the same condition. Apparently high-complexity subjects failed to demonstrate the same improvement in their response latency scores because they attended to the new and inconsistent information about the self. Once available in memory, the inconsistent information slowed down the subsequent self-evaluative judgments. The high-complexity individual could no longer quickly and efficiency endorse the intelligent trait adjectives as highly self-descriptive. Rather the individual had to engage in more thought before the judgments could be made.

The fact that low-complexity subjects in the failure condition experienced a marked decrease in response latency time between time 1 and time 2 suggests that the low complexity subjects did not take in the new and inconsistent information. In this case the lack of a rich and differentiated structure limited the individual's ability to attend to and encode the new information. Consequently, when subsequent self-descriptiveness judgments were made, no additional processing time was needed to reconcile the new and inconsistent information with the established self-view.

The pattern of changes in self-descriptiveness ratings provides additional insight into processing differences between the two groups. The one clear finding in this analysis is that high-complexity subjects experienced less change in their self-descriptiveness ratings than the low-complexity subjects regardless of the feedback condition. Even though the response latency measures indicate that the high-complexity subjects did take in and consider the inconsistent information, the pattern of findings for the selfdescriptiveness ratings suggests that the individual's generalized view of the self in the domain was not measurably altered by this single piece of information.

When taken together these data support the view that a highly complex self-schema may be considered a stable but flexible cognitive structure. The schema may be considered stable in that the general view of the self is not capriciously altered each time a new and inconsistent piece of selfrelevant information is encountered. This stability is, however, not maintained by rigidly blocking out or ignoring inconsistent feedback. Rather, the individual with a highly complex self-schema has the capacity to consider and take in a broader range of information about the self and in 
doing so creates a means by which the scheme can be further elaborated, modified, or changed.

The fact that low-complexity subjects in this study experienced more change between time 1 and time 2 in their self-descriptiveness ratings than the high-complexity group is consistent with the results of previous work that suggests that low-complexity subjects react to new information about a target with a more extreme evaluation (Linville, 1982, 1985; Linville \& Jones, 1980). The one finding that is surprising is that low-complexity subjects tended to describe themselves more positively after the failure feedback was given. Although the response latency data suggest that low-complexity subjects did not take in the failure feedback, the self-description data suggest that the feedback did trigger a defensive reaction. The positive increase in self-descriptiveness scores suggests that low-complexity subjects did not simply ignore the inconsistent feedback but more actively sought to discount the feedback by reaffirming the strongly positive view of the self in the domain. In this way stability of the self is maintained, but flexibility to consider new information about the self is compromised.

The idea that complexity of the self-concept plays an important role in shaping responses to challenging feedback may help to clarify results of studies reported by Power and colleagues (Power \& Brewin, 1990; Power, Brewin, Stuessy, \& Mahony, 1991). In the 1990 study, Power and Brewin found that exposure to self-esteem threatening and survival-threatening stimuli (i.e., written statements such as, "You fail to get the job you want" or "You are assaulted") led to a tendency for subjects to endorse more positive adjectives as self-descriptive at a faster rate than negative adjectives. In the 1991 study, exposure to basic negative emotion words such as sad, fear, and anger led to a similar pattern of findings. The results of this current study suggest that the trends in Power and colleagues' findings perhaps could be clarified by taking into account the level of complexity of the self-structure. Rather than viewing defensive self-affirmation as universal, it may be more appropriately seen as a type of response linked to a specific organization of the self-structure. Furthermore, the fact that survival-threatening stimuli and negative emotion words also triggered the self-affirmation process raises the possibility that the processing differences observed in low-complexity subjects may be set in motion by a broad range of threats to the self that go beyond direct challenges to self-esteem. Additional research is needed to clarify the nature of the stimuli and the level of involvement of the self that is necessary for the defensive response to occur.

Of course at this point in time, the long-term consequences of the observed processing differences are unknown. Although one can speculate that the high-complexity individual's ability to take in and consider new information is adaptive and represents a type of flexibility in thinking nec- 
essary to maintain a realistic and current conception of oneself, an alternative hypothesis is equally plausible. Given that a variety of cognitive distortions including the maintenance of an unrealistically positive view of one's self and one's future are associated with higher levels of mental and physical health (see Taylor \& Brown, 1988), it is possible that the processing style of the low-complexity individual is highly functional (see Powers et al., 1991). From this view the ability to actively resist discomforting feedback and maintain a highly positive self-view may be seen as an important self-regulatory function that contributes to sustained optimism and a positive state of well-being. Additional studies are needed to replicate the findings of this study and to examine the short- and long-term cognitive and affective consequences of these different information processing styles.

\section{REFERENCES}

Ammons, R. B., \& Ammons, C. H. (1962). The Quick Test: Provisional manual. Psychological Reports, 11, 111-116.

Bieri, J. (1955). Cognitive complexity-simplicity and predictive behavior. Journal of Abnormal Psychology, 51, 263-268.

Burleson, B. (1987). Cognitive complexity. In J. C. McCroskey \& J. A. Daly (Eds.), Personality and interpersonal communication (pp. 305-349). New York: Sage.

Crockett, W. (1965). Cognitive complexity and impression formation. In B. Maher (Ed.), Progress in experimental personality research (Vol. 2, pp. 47-90). New York: Academic Press.

Greenwald, A. G. (1980). The totalitarian ego fabrication and revision of personal history. American Psychologist, 35, 603-617.

Greenwald, A. G., \& Pratkanis, A. R. (1984). The self. In R. S. Wyer \& T. K. Srull (Eds.), Handbook of social cognition (Vol. 3, pp. 129-178). Hillsdale, NJ: Erlbaum.

Hastie, R. (1981). Schematic principles in human memory. In E. T. Higgins, C. P. Herman, \& M. P. Zanna (Eds.), Social cognition: The Ontario Symposium (pp. 39-88). Hillsdale, NJ: Erlbaum.

Leventhal, H. (1957). Cognitive processes and interpersonal predictions. Journal of Abnormal and Social Psychology, 55, 176-180.

Lewicki, P. (1986). Nonconscious social information processing. New York: Academic Press.

Linville, P. (1982). Affective consequences of complexity regarding the self and others. In $M$. S. Clark \& S. T. Fiske (Eds.), Affect and cognition (pp. 79-109). Hillsdale, NJ: Erlbaum.

Linville, P. (1985). Self-complexity and affective extremity: Don't put all your eggs in one cognitive basket. Social Cognition, 3, 94-120.

Linville, P. (1987). Self-complexity as a cognitive buffer against stress-related illness and depression. Journal of Personality and Social Psychology, 52, 663-676.

Linville, P., \& Jones, E. (1980). Polarized appraisals of out-group members. Journal of Personality and Social Psychology, 38, 689-703.

Markus, H. (1977). Self-schemata and the processing of information about the self. Journal of Personality and Social Psychology, 35, 63-78.

Markus, H., \& Sentis, K. (1982). The self in social information processing. In J. Suls (Ed.), Psychological perspectives on the self (Vol. 1, pp. 41-70). Hillsdale, NJ: Erlbaum.

Mayo, C., \& Crockett, W. (1964). Cognitive complexity and primacy-recently effects in impression formation. Journal of Abnormal and Social Psychology, 68, 335-338. 
Meltzer, B., Crockett, W., \& Rosenkrantz, P. (1966). Cognitive complexity, value congruity, and the integration of potentially incompatible information in impressions of others. Journal of Personality and Social Psychology, 4, 338-343.

O'Keefe, D., \& Sypher, H. (1981). Cognitive complexity and the relationship of cognitive complexity to communication. Human Communication Research, 8, 72-92.

Power, M. J., \& Brewin, C. R. (1990). Self-esteem regulation in an emotional priming task. Cognition and Emotion, 4, 39-51.

Power, M. J., Brewin, C. R., Stuessy, A., \& Mahony, T. (1991). The emotional priming task: Results from a student population. Cognitive Therapy and Research, 15, 21-31.

Rosenberg, M. (1965). Society and the adolescent self-image. Lawrenceville, NJ: Princeton University Press.

Scott, W., Osgood, D. W., \& Peterson, C. (1979). Cognitive structure: Theory and measurement of individual difference. New York: John Wiley.

Swann, W. B., \& Read, S. J. (1981). Self-verification processes: How we sustain our self-conceptions. Journal of Experimental Social Psychology, 17, 351-372.

Taylor, S. E., \& Brown, J. D. (1988). Illusion and well-being: A social psychological perspective on mental health. Psychological Bulletin, 103, 193-210.

Taylor, S. E., \& Crocker, J. (1981). Schematic bases of social information processing. In E. T. Higgins, C. P. Herman, \& M. P. Zanna (Eds.), Social cognition: The Ontario Symposium (pp. 39-88). Hillsdale, NJ: Erlbaum.

Zajonc, R. B. (1954). Cognitive structure and cognitive tuning. Unpublished doctoral dissertation, University of Michigan, Ann Arbor.

Zajonc, R. B. (1960). The process of cognitive tuning in communication. Journal of Abnormal and Social Psychology, 61, 159-167. 